Ana Helena Dias Pereira dos Santos Ulbrich

Catiane Tiecher Cusinato

Roberta da Silva Guahyba

Hospital Nossa Senhora da Conceição

Como citar este artigo:

Ulbrich AHDPS, Cusinato CT e Guahyba RS. Medicamentos Potencialmente Inapropriados (MIPS) Idosos: Prevalência em um Hospital Terciário do Brasil. Rev. Bras. Farm. Hosp. Serv. Saúde 8(3): 14-18, 2017. Doi: $10.30968 /$ rbfhss.2017.083.003

Recebido: 24/02/17

Revisado: 19/06/17

Aceito: 05/08/17

Autor Correspondente: Ana Helena Dias Pereira dos Santos Ulbrich Hospital Nossa Senhora da Conceição E-mail: anahelena@gemeos.org

\section{MEDICAMENTOS POTENCIALMENTE INAPROPRIADOS (MPIS) PARA IDOSOS: PREVALÊNCIA EM UM HOSPITAL TERCIÁRIO DO BRASIL}

POTENTIALLY INAPPROPRIATE MEDICATIONS (PIMS) IN THE ELDERLY PATIENT: PREVALENCE STUDY IN A TERTIARY HOSPITAL IN BRAZIL

\author{
MEDICAMENTOS POTENCIALMENTE INAPROPIADOS (MPI) EN \\ ANCIANOS: LA PREVALENCIA EN UN HOSPITAL TERCER NIVEL EN BRASIL
}

\section{RESUMO}

Introdução: A alta prevalência de doenças em idosos é uma das responsáveis pela elevada utilização de medicamentos. Várias estratégias foram desenvolvidas para reduzir o impacto das prescrições na população idosa, entre elas, a detecção de Medicamentos Potencialmente Inapropriados (MPI). Os MPI estão associados ao aumento de morbidades, mortalidade e gastos de recursos em saúde. Existe uma grande variedade de estudos descrevendo a prevalência de MPI prescritos para idosos, sendo o Critério de Beers o mais utilizado para determinar a seleção dos medicamentos a serem considerados potencialmente inapropriados. Objetivos: Verificar a prevalência de Medicamentos Potencialmente Inapropriados (MPI) prescritos para idosos em um Hospital Terciário. Metodologia: Foi realizada uma análise retrospectiva dos pacientes idosos admitidos entre janeiro de 2014 e dezembro de 2014. Os medicamentos potencialmente inapropriados foram avaliados nas prescrições dos pacientes idosos através dos Critérios de Beers de 2012 e a Lista de Laroche de 2007. Resultados: Dos 695 pacientes idosos, 639 (91,9\%), segundo o critério de Beers 2012, e 41,2\%, segundo os Critérios de Laroche, tiveram pelo menos um MPI prescrito em 2014. Os MPIs mais prescritos foram metoclopramida (54,2\%), diazepam (9,7\%) e hioscina/escopolamina $(7,1 \%)$ segundo os Critérios de Beers. Conclusões: Este estudo mostra uma grande prevalência de MPIs prescritos para idosos, necessitando de políticas de redução e racionalização da prescrição destes medicamentos.

Palavras-chave: Medicamentos Potencialmente Inapropriados, Idoso, Prescrição Inadequada, Segurança do Paciente, Polifarmácia, Critérios de Beers

\begin{abstract}
Introduction: The high prevalence of diseases in the elderly person is responsible for the high use of medications. Several strategies have been developed to reduce the impact of prescriptions on the elderly population, including the detection of Potentially Inappropriate Medications (PIM). PIM is associated with increased morbidity, mortality, and health resource expenditures. There is a wide variety of studies describing the prevalence of PIM prescribed for the elderly person, with Beers' Criteria being the most used to determine the selection of drugs to be considered potentially inappropriate. Goals: To evaluate the prevalence of potentially inappropriate medications (PIMs) prescribed in elderly inpatients in a tertiary hospital. Methods: Retrospective analysis was performed for cases of older patients admitted between January 2014 and December 2014. Prescriptions were assessed for the use of potentially inappropriate medications in geriatric patients by using American Geriatric Society Beer's criteria (2012) a Laroche List (2007). Results: Of the 695 elderly people in the study population, 639 (91,9\%) received at least one PIM prescription in 2014. The most commonly prescribed PIM were metoclopramide (54,2\%), diazepam (9,7\%) and scopolamine/hyoscine (7,1\%) Conclusions: This study shows a high prevalence of PIM prescriptions for the elderly population, requiring politics for reduction and rationalization of prescribing these drugs.
\end{abstract}

Keywords: Potentially Inappropriate Medication, Aged, Inappropriate Prescribing, Patient Safety, Polypharmacy, Beers Criteria.

\section{RESUMEN}

Introducción: La alta prevalencia de enfermedades en los ancianos es una de las responsables de la elevada utilización de medicamentos. Se desarrollaron varias estrategias para reducir el impacto de las prescripciones en la población anciana, entre ellas, la detección de Medicamentos Potencialmente Inapropiados (MPI).Los MPI están asociados al aumento de morbilidad, mortalidad y gastos de recursos en salud. Hay una gran variedad de estudios que describen la prevalencia de MPI prescritos para los ancianos, siendo el Criterio de Beers el más utilizado para determinar la selección de los medicamentos a ser considerados potencialmente inapropiados. Objetivos: Conocer la prevalencia de prescripción de fármacos potencialmente inapropiados prescritos a pacientes ancianos en un hospital de tercer nivel. Métodos: Un estudio retrospectivo de los pacientes ancianos ingresados entre enero de 2014 al diciembre de 2014. Los medicamentos potencialmente inapropiados fueron evaluados en los expedientes de los pacientes ancianos de acuerdo con los criterios de Beer (2012) e la lista de Laroche (2007). Resultados: De los 695 pacientesidosos, 639 (91,9\%), según el criterio de Beers de 2012,y 41,2\%, de acuerdo con criterios de Laroche, tenían al menos un MPI prescrito en 2014.Los MPI más prescritos fueron metoclopramida (54,2\%), diazepam (9,7\%) y escopolamina (7,1\%) de acuerdo con los criterios de Beers. Conclusiones: Este estudio muestra una alta prevalencia de prescripción de los MPI para los ancianos, que requiere pollíticas de reducción y racionalización de la prescripción de estos fármacos.

Palabras clave: Medicamentos Potencialmente Inapropiados, Anciano, Prescripción Inadecuada, Seguridad del Paciente, Polifarmacia, Criterios de Beers. 


\section{INTRODUÇÃO}

O envelhecimento populacional é uma realidade em todo o mundo. A Organização Mundial da Saúde classifica como idosa a pessoa com 65 ou mais anos de idade em países desenvolvidos e com 60 ou mais anos de idade em países em desenvolvimento. A população com 60 anos ou mais no Brasil está crescendo e estima-se que alcançará 33,4 milhões em 2025 segundo a Organização Mundial da Saúde ${ }^{1}$.

Aalta prevalência de doenças emidososéuma das responsáveis pela elevada utilização de medicamentos, já que a intervenção terapêutica mais freqüente é a prescrição destes. $\mathrm{O}$ número de medicamentos, a complexidade dos regimes terapêuticos, especialmente na vigência de co-morbidades e as alterações farmacocinéticas e farmacodinâmicas inerentes ao processo de envelhecimento são elementos que aumentam a vulnerabilidade desse grupo etário aos eventos adversos, seja por reações adversas, seja por interações medicamentosas².

A preocupação com o impacto das prescrições na população idosa tem levado à criação de várias estratégias para lidar com esse fenômeno, entre elas, a detecção de Medicamentos Potencialmente Inapropriados (MPI). Medicamentos são potencialmente inapropriados quando o risco de provocar efeitos adversos é superior aos benefícios em idosos ${ }^{3}$. Os MPI também estão associados ao aumento de morbidades, mortalidade e gastos de recursos em saúde. Neste contexto, algumas categorias de medicamentos passaram a ser consideradas impróprias para o idoso, seja por falta de eficácia terapêutica, seja por um risco aumentado de efeitos adversos que superam os benefícios. Parcela significativa dos eventos adversos que ocorrem com os idosos pode ser prevenida no momento da prescrição, através do uso de listas de MPI para idosos, servindo como auxílio na prática clínica. ${ }^{3}$ Várias ferramentas que classificam os MPI, criadas por grupos de especialistas na área através de revisões da literatura médica, já foram publicadas nas duas últimas décadas, como Critério de Beers ${ }^{4}, 5$, BeerFick $^{6}$ e sua atualização ${ }^{7}$, Screening Tool of Older persons' Potentially inappropriate Prescriptions (STOPP) ${ }^{8}$, Improving Prescribing in the Elderly Too (IPET) ${ }^{9}$, Lista francesa de Laroche ${ }^{10}$, entre outros.

Existe uma grande variedade de estudos descrevendo a prevalência de MPI prescritos para idosos, sendo o Critério de Beers o mais utilizado para determinar a seleção dos medicamentos a serem considerados potencialmente inapropriados. Os resultados de prevalência dos MPI prescritos nos estudos apresentados na literatura são altos, mas dependem do método usado para avaliar o uso impróprio. Além da diversidade de métodos, outros fatores, tais como sexo do paciente, idade e número de medicamentos utilizados concomitantemente parecem ter influência nas estimativas dos $\mathrm{MPI}^{11}$. Além destes fatores, a complexidade do local onde o estudo é realizado também influencia no resultado da prevalência, já que muitas vezes em hospitais o uso de MPIs se faz necessário em algumas situações, contrastando com a atenção primária. O uso dos MPIs varia muito entre os diversos países, já que os medicamentos disponíveis para prescrição são distintos, tendo resultados de prevalência de $25,5 \%$ a 98,2\% ${ }^{12-16}$. Um estudo realizado em um hospital terciário no Brasil apresentou uma alta prevalência do uso dos MPIs, tendo 95,5\% dos idosos com pelo menos um MPI prescrito de acordo com o Critério de Beer (versão 2012), sendo a metoclopramida, o ácido acetilsalicílico e o cetoprofeno os MPI mais prescritos ${ }^{14}$.

Estudos mostram que o fornecimento de informações sobre o uso inadequado de medicamentos, sistema de suporte automatizado e o envolvimento de equipes multidisciplinares são necessários para melhorar o atendimento de pacientes com regime de medicamentos complexos. Uma abordagem abrangente incluindo mais de um método para melhorar a prescrição inadequada em idosos deve ser desenvolvida ${ }^{11,12,13}$.

Este estudo pretende determinar a prevalência de MPI presentes nas prescrições de idosos internados no Hospital Nossa Senhora da Conceição de Porto Alegre, região sul do Brasil.

\section{METODOLOGIA}

Trata-se de um estudo transversal, com utilização de dados secundários obtidos através do prontuário eletrônico disponível no sistema informatizado do hospital. A população do estudo foi composta por pacientes com idade igual ou superior a 60 anos que foram admitidos entre janeiro e dezembro de 2014 no Hospital Nossa Senhora da Conceição, hospital público terciário de 843 leitos. A coleta de dados foi executada pelo Serviço de Farmácia. Foram excluídos do estudo pacientes idosos cujo período de internação era inferior a 5 dias ou que continuavam internados no momento da coleta de dados. Somente a primeira internação foi considerada nos pacientes que tiveram mais de uma hospitalização durante o ano de 2014.

Os dados das prescrições médicas foram coletados em três momentos diferentes: na admissão (prescrição do primeiro dia de internação); durante a hospitalização (prescrição do terceiro dia), e na alta hospitalar (última prescrição no hospital).

As variáveis do instrumento de pesquisa foram do tipo categóricas (sexo, razão principal da internação, presença de MPI, área médica e polifarmácia) e contínuas (idade, número total de medicamentos prescritos, número total de MPI prescritos, doses e índice de comorbidade de Charlson).

No ano de 2014 foram internados aproximadamente 4700 idosos com no mínimo 5 dias de internação. Considerando um percentual de MPI em idosos de 50\%, com erro aceitável de 5\%, um efeito de delineamento de 2,0, o tamanho da amostra estimado foi 711 para o intervalo de confiança de $95 \%$.

A presença ou não de polifarmácia foi definida pela existência de cinco ou mais medicamentos prescritos para o paciente ${ }^{14,21}$.

A avaliação foi realizada através da utilização dos Critérios de Beers versão 2012 e lista de Laroche, considerando-se apenas aqueles medicamentos que independem de diagnóstico e são padronizados no hospital. Assim como outros estudos, a lista foi adaptada de acordo com os medicamentos disponíveis no país. Foram incluídos na lista por serem também pertencentes às classes descritas: flunitrazepam (benzodiazepínico), midazolam (benzodiazepínico) e levomepromazina (antipsicótico convencional) na lista de Beers e clonazepam (benzodiazepínico de longa ação) na lista de Laroche. A Insulina de escala móvel não foi considerada. Os medicamentos que são considerados inapropriados quando superior a determinada dose tiveram sua dose avaliada para ser considerado como MPI.

A análise dos resultados foi realizada utilizando-se o programa SPSS versão 18.

Este estudo foi aprovado pelo Comitê de Ética em Pesquisa do Grupo Hospitalar Conceição em 11 de março de 2015 sob o registro 15030.

\section{RESULTADOS}

Foram avaliados os medicamentos das prescrições em três momentos (admissão, internação e alta/óbito), sendo que na admissão, 695 pacientes tiveram seus medicamentos avaliados, totalizando 6098 medicamentos prescritos (média 8,8 - DP 3,7). No terceiro dia de internação (momento que representa a internação) também foram avaliadas as prescrições de 695 pacientes, tendo 6956 medicamentos prescritos (média 10,0 - DP 3,6). Já na última prescrição do paciente (antes da alta ou óbito), foram analisados 692 pacientes que tiveram 7670 medicamentos prescritos (média 11,1-DP 4,1).

As características dos participantes do estudo são mostradas na Tabela 1. Os idosos participantes foram $51,2 \%$ masculinos, de idade entre 60 e 94 anos com uma média de 71,79 anos (Desvio Padrão $(\mathrm{DP})=8,2$ ).

Dos 695 pacientes avaliados, 501 tiveram o índice de comorbidade de Charlson preenchido, resultando em uma média (DP) de 3,25 (2,46) e variando entre 1 e 14.

Dentre os idosos avaliados neste estudo, as doenças mais comuns que motivaram a internação foram as relacionadas ao sistema circulatório, neoplasias e doenças do sistema digestivo. As comorbidades mais frequentes foram neoplasias, hipertensão e diabetes. 
Tabela 1. Características demográficas e clínicas da população do estudo $(n=695)$

\begin{tabular}{|c|c|c|c|}
\hline & & $\mathbf{n}$ & $\%$ \\
\hline \multirow[t]{2}{*}{ Sexo } & Masculino & 356 & 51,2 \\
\hline & Feminino & 339 & 48,8 \\
\hline \multirow[t]{3}{*}{ Idade } & $60-69$ & 309 & 44,4 \\
\hline & $70-79$ & 257 & 36,9 \\
\hline & $>80$ & 129 & 18,6 \\
\hline \multirow[t]{3}{*}{ Raça } & Caucasianos & 617 & 88,6 \\
\hline & Afrodescendentes & 73 & 10,5 \\
\hline & Outros & 5 & 0,7 \\
\hline \multirow[t]{3}{*}{ Comorbidade } & Índice de Charlson: 1 - 3 & 341 & 68,1 \\
\hline & Índice de Charlson: 4 - 8 & 143 & 28,5 \\
\hline & Índice de Charlson: 9 - 16 & 17 & 3,4 \\
\hline \multirow[t]{2}{*}{ Polifarmácia } & 1-4 medicamentos & 23 & 3,3 \\
\hline & 5 ou mais medicamentos & 672 & 96,7 \\
\hline \multirow[t]{6}{*}{ Serviço na alta } & Especialidades Médicas & 252 & 36,3 \\
\hline & Medicina Geral & 166 & 23,9 \\
\hline & Especialidades Cirúrgicas & 125 & 18,0 \\
\hline & Cirurgia Geral & 63 & 9,1 \\
\hline & Emergência & 47 & 6,8 \\
\hline & Unidade de Terapia Intensiva & 42 & 6 \\
\hline \multirow[t]{8}{*}{ CID principal } & Doenças do Sistema Circulatório & 179 & 25,8 \\
\hline & Neoplasias & 138 & 19,9 \\
\hline & Doenças do Sistema Digestivo & 85 & 12,2 \\
\hline & Doenças do Sistema Respiratório & 67 & 9,6 \\
\hline & Doenças do Sistema Geniturinário & 57 & 8,2 \\
\hline & $\begin{array}{l}\text { Sinais, sintomas e achados clínicos e laboratoriai } \\
\text { anormais }\end{array}$ & 53 & 7,6 \\
\hline & Doenças parasitárias e infecciosas & 45 & 6,5 \\
\hline & Doenças endócrinas, nutricional e metabólica. & 21 & 3,0 \\
\hline \multirow{5}{*}{$\begin{array}{l}\text { Comorbidades } \\
\text { mais frequentes }\end{array}$} & Neoplasia maligna & 191 & 27,5 \\
\hline & Hipertensão & 124 & 17,8 \\
\hline & Diabetes mellitus & 112 & 16,1 \\
\hline & Desordem de pele ou subcutânea & 84 & 12,1 \\
\hline & Doenças do sistema circulatório & 105 & 15,2 \\
\hline
\end{tabular}

Nossos dados mostraram que dos 695 pacientes avaliados, 639 (91,9\%) e $268(41,2 \%)$ tinham prescrito pelo menos um medicamento potencialmente inapropriado segundo a lista de Beers e lista de Laroche, respectivamente. Já quando analisada a prevalência de 2 ou mais medicamentos potencialmente inapropriados, de acordo com a lista de Beers foram 45,3\% e de acordo com a lista de Laroche, 10,2\%. Além disso, a média de MPIs por paciente foi de 1,5496 (Desvio Padrão $(D P)=0,93294)$ para a lista de Beers e $0,534(\mathrm{DP}=0,7350)$ para a lista de Laroche.

O número total e percentual de pacientes expostos a pelo menos um MPI e 2 ou mais MPIs, determinado através de duas ferramentas são apresentados na Tabela 2.

Os cinco medicamentos mais prescritos foram: metoclopramida, dipirona, heparina, omeprazol e morfina. Além disso, aproximadamente 80\% dos pacientes que tiveram a sua prescrição analisada tinham prescrito dipirona, heparina e metoclopramida, sugerindo um padrão de prescrição.

Dentre o total de medicamentos prescritos durante a internação, 15,48\% eram MPI segundo a lista de Beers e 5,33\%, segundo a lista de Laroche.

Quando utilizado a lista de MPIs segundo os critérios de Beers, os MPIs mais frequentemente prescritos foram metoclopramida, diazepam e escopolamina (Tabela 3). Apesar do ácido acetilsalićlico estar entre os medicamentos mais prescritos, a dose prescrita é menor que $325 \mathrm{mg}$, não sendo considerada inapropriada.
Tabela 2. Comparação do número de MPIs prescritos por paciente em cada estágio da internação

\begin{tabular}{|c|c|c|c|c|c|c|c|c|}
\hline \multirow[t]{2}{*}{ Ferramenta } & \multirow[t]{2}{*}{$\begin{array}{l}\text { Estágio da } \\
\text { internação }\end{array}$} & \multirow{2}{*}{$\begin{array}{c}\text { Número } \\
\text { de } \\
\text { pacientes }\end{array}$} & \multirow{2}{*}{$\begin{array}{l}\text { Média } \\
\text { MPI por } \\
\text { paciente } \\
\text { (DP) }\end{array}$} & \multirow{2}{*}{$\begin{array}{l}\text { Máximo } \\
\text { MPI por } \\
\text { paciente }\end{array}$} & \multicolumn{2}{|c|}{$\begin{array}{c}\text { Pelo menos } \\
1 \mathrm{MPI}\end{array}$} & \multicolumn{2}{|c|}{$\geq 2$ MPIs } \\
\hline & & & & & $\mathrm{n}$ & $\%$ & $\mathrm{n}$ & $\%$ \\
\hline \multirow[t]{3}{*}{ Beers } & Admissão & 695 & $\begin{array}{c}1,401 \\
(08894)\end{array}$ & 5,00 & 607 & 87,3 & 288 & 41,4 \\
\hline & Internação & 695 & $\begin{array}{c}1,550 \\
(0,9329)\end{array}$ & 5,00 & 639 & 91,9 & 315 & 45,3 \\
\hline & $\begin{array}{l}\text { Última } \\
\text { prescrição }\end{array}$ & 692 & $\begin{array}{c}1,746 \\
(1,0031)\end{array}$ & 6,00 & 650 & 93,5 & 376 & 54,1 \\
\hline \multirow[t]{3}{*}{ Laroche } & Admissão & 695 & $\begin{array}{c}0,470 \\
(0,6565)\end{array}$ & 3,00 & 268 & 38,6 & 55 & 7,9 \\
\hline & Internação & 695 & $\begin{array}{c}0,534 \\
(0,7350)\end{array}$ & 4,00 & 268 & 41,2 & 71 & 10,2 \\
\hline & $\begin{array}{l}\text { Última } \\
\text { prescrição }\end{array}$ & 692 & $\begin{array}{c}0,666 \\
(0,8488)\end{array}$ & 4,00 & 324 & 46,8 & 106 & 15,3 \\
\hline
\end{tabular}

Tabela 3. MPIs mais frequentes segundo a lista de Beers, (\%)

\begin{tabular}{|c|c|c|c|}
\hline MPI & Classe terapêutica & $\begin{array}{l}\text { Qualidade da } \\
\text { evidência }\end{array}$ & $\%$ \\
\hline Metoclopramida & Gastrointestinal & Moderada & 54,2 \\
\hline Diazepam & Benzodiazepínico & Alta & 9,7 \\
\hline $\begin{array}{l}\text { Escopolamina / } \\
\text { Hioscina }\end{array}$ & Antiespasmódico & Moderada & 7,1 \\
\hline Clonidina & Alfa-agonista de ação central & Baixa & 4,7 \\
\hline Haloperidol & Antipsicótico convencional & Moderada & 4,0 \\
\hline Clonazepam & Benzodiazepínico & Alta & 3,3 \\
\hline Midazolam & Benzodiazepínico & Alta & 3,2 \\
\hline Doxazosina & Cardiovascular - alfabloqueador & Moderada & 1,8 \\
\hline Amitriptilina & $\begin{array}{l}\text { Antidepressivo tricíclico } \\
\text { terciário }\end{array}$ & Alta & 1,7 \\
\hline Amiodarona & Antiarrítmico & Alta & 1,6 \\
\hline $\begin{array}{l}\text { Digoxina } \\
>0.125 \mathrm{mg} / \text { dia }\end{array}$ & Cardiovascular & Moderada & 1,4 \\
\hline Óleo Mineral & Gastrointestinal & Moderada & 1,4 \\
\hline Cetoprofeno & $\begin{array}{l}\text { Anti-inflamatório não esteroidal } \\
\text { COX-não seletivo }\end{array}$ & Moderada & 1,3 \\
\hline Prometazina & $\begin{array}{l}\text { Anticolinérgico } \\
\text { (Antihistamínico de primeira } \\
\text { geração) }\end{array}$ & Alta & 1,1 \\
\hline Risperidona & $\begin{array}{l}\text { Antipsicótico atípico } \\
\text { (Segunda geração) }\end{array}$ & Moderada & 0,7 \\
\hline Clorpromazina & Antipsicótico convencional & Moderada & 0,6 \\
\hline Fenobarbital & Barbitúrico & Alta & 0,6 \\
\hline $\begin{array}{l}\text { Espironolactona } \\
>25 \mathrm{mg} / \text { dia }\end{array}$ & Cardiovascular & Moderada & 0,5 \\
\hline Dexclorfeniramina & $\begin{array}{l}\text { Anticolinérgico } \\
\text { (Antihistamínico de primeira } \\
\text { geração) }\end{array}$ & Moderada & 0,4 \\
\hline Diclofenaco & $\begin{array}{l}\text { Anti-inflamatório não esteroidal } \\
\text { COX-não seletivo }\end{array}$ & Moderada & 0,3 \\
\hline Nifedipina & Cardiovascular & Alta & 0,3 \\
\hline Olanzapina & Antipsicótico atípico & Moderada & 0,2 \\
\hline Flunitrazepam & Benzodiazepínico & Alta & 0,1 \\
\hline
\end{tabular}


Quando utilizada a lista de MPIs segundo os critérios de Laroche, os MPIs mais frequentemente prescritos foram diazepam, escopolamina e clonidina (Tabela 4).

Tabela 4. MPIs mais frequentes segundo a lista de Laroche (\%)

\begin{tabular}{|c|c|c|c|}
\hline MPI & Classe Terapêutica & Frequência & $\%$ \\
\hline Diazepam & Benzodiazepínico de longa ação & 104 & 28,0 \\
\hline $\begin{array}{l}\text { Escopolamina / } \\
\text { Hioscina }\end{array}$ & Antiespasmódico & 77 & 20,8 \\
\hline Clonidina & Antihipertensivo de ação central & 51 & 13,7 \\
\hline Clonazepam & Benzodiazepínico de longa ação & 36 & 9,7 \\
\hline Bisacodil & Estimulante laxativo & 29 & 7,8 \\
\hline Amitriptilina & Anticolinérgico & 18 & 4,9 \\
\hline $\begin{array}{l}\text { Digoxina } \\
(>0,125 \mathrm{mcg})\end{array}$ & Antiarrítmico & 15 & 4,0 \\
\hline Óleo Mineral & Estimulante laxativo & 15 & 4,0 \\
\hline Prometazina & Anticolinérgico & 12 & 3,2 \\
\hline Clorpromazina & Anticolinérgico & 6 & 16 \\
\hline Dexclorfeniramina & Anticolinérgico & 4 & 1,1 \\
\hline Nifedipine & $\begin{array}{l}\text { Antihipertensivo - Bloqueador de } \\
\text { canal de cálcio }\end{array}$ & 3 & 0,8 \\
\hline Flunitrazepam & Benzodiazepínico de longa ação & 1 & 0,3 \\
\hline
\end{tabular}

Apesar de não haver diferença significativa quanto à presença de MPI entre as faixas de idade quando usada a lista de Beers, houve diferença significativa (Teste ANOVA) na presença de MPI quando utilizada a lista de Laroche, sendo que os idosos da faixa de 60 a 69 tiveram mais MPIs prescritos.

A média de MPI segundo ambos critérios foi maior entre os idosos com polifarmácia que entre os idosos sem polifarmácia $-P<0,001$ (Tabela 5).

Tabela 5. Correlação entre a polifarmácia e a média de MPIs.

\begin{tabular}{lccccc}
\hline & \multicolumn{5}{c}{ Polifarmácia } \\
\cline { 2 - 5 } Ferramenta & \multicolumn{3}{c}{ Sim } \\
\cline { 2 - 5 } & Média de MPI & DP & Média de MPI & DP \\
\hline Beers & 1,5714 & 0,937 & 0,913 & 0,515 & $<0,001$ \\
Laroche & 0,549 & 0,741 & 0,087 & 0,288 & $<0,001$ \\
\hline
\end{tabular}

O gênero, tempo de permanência e o índice de comorbidade de Charlson não apresentaram influência na prescrição de MPIs.

\section{DISCUSSÃO}

Uma grande quantidade de prescrições com MPIs para idosos tem sido observada em diversos estudos, ${ }^{14,15,16,20}$ o que mostra a necessidade de melhorar essa situação. A comparação entre resultados dos estudos é dificultada pelas diferentes ferramentas utilizadas, formas de coleta distintas, medicamentos disponíveis para prescrição e características das populações. Apesar destas limitações, a prevalência de MPIs encontrados nas prescrições dos idosos no hospital usando o critério de Beers é alta $(91,9 \%)$ quando comparada com outros estudos que usam o mesmo critério em países como Nigéria - $25,5 \%{ }^{12}$, e semelhante quando comparada com estudos realizados no Canadá $(92 \%)^{15}$, Índia $(87,3 \%)^{15} \mathrm{e}$ outros estudos realizados no Brasil $\left(95,5 \%{ }^{14}\right.$ e $\left.98,2 \%{ }^{16}\right)$.
Os estudos realizados no Brasil ${ }^{14,16}$, assim como este estudo, tiveram a metoclopramida como mais prevalente MPI prescrito, estando também entre os MPIs mais prescritos os benzodiazepínicos. Importante a discussão com as equipes médicas sobre a inclusão da metoclopramida como medicamento padrão de prescrição, mesmo sendo medicamento prescrito como "se necessário".

$\mathrm{Na}$ Índia, o medicamento que eleva a prevalência de MPI na prescrição de idosos, assim como no Brasil ${ }^{16}$, é a metoclopramida, usada para gastroparesia e como antiemético de baixo custo. A inclusão do medicamento na lista do Critério de Beers de 2012 fez com que o resultado da prevalência aumentasse quando comparada com a versão de 2002. Apesar de aprovado o uso para tratamento da gastroparesia, seu uso é considerado inapropriado em função da possibilidade de reações adversas extrapiramidais. $\mathrm{O}$ tratamento prolongado com metoclopramida também pode causar reações adversas sérias como discinesia tardia persistente. ${ }^{15}$ Apesar do medicamento mais prescrito ser a metoclopramida, quando se trata de efeito adverso relacionado ao medicamento, a maior parte dos estudos apresenta poucos eventos ou nenhum relacionado à metoclopramida, tendo maior evidências de eventos relacionados aos benzodiazepínicos. $^{16}$

Os benzodiazepínicos são prescritos principalmente para o tratamento de insônia e ansiedade, podendo afetar a função cognitiva. Seu efeito sedativo é um fator de risco para quedas e fraturas ${ }^{16,21,17}$. $\mathrm{O}$ aumento no risco de quedas e fraturas entre idosos usuários de benzodiazepínicos tem sido atribuído a duas propriedades desses medicamentos: atividade sedativa e bloqueio $\alpha$-adrenérgico. ${ }^{18}$ A primeira seria responsável por alterações psicomotoras, enquanto a segunda aumentaria a probabilidade de hipotensão postural. Os agentes hipnótico-sedativos de meia-vida longa, quando utilizados em doses clinicamente efetivas, podem causar sedação residual durante o dia entre os idosos. Com isso, esses indivíduos estão mais sujeitos a apresentar tonturas, ataxia, confusão, levando ao risco aumentado de quedas. A redução da massa muscular e da densidade óssea acarreta uma probabilidade aumentada de que tais quedas resultem em fraturas. ${ }^{23}$

Quando se compara a prevalência de MPIs prescritos para idosos de acordo com as diferentes ferramentas (Beers - 91,9\%, Laroche $41,2 \%$ ), percebe-se uma grande diferença que associamos a presença da metoclopramida na lista de Beers e a ausência na lista de Laroche. Isso explica a semelhança entre a prevalência de prescrições com pelos menos 1 MPI pela lista de Laroche $(41,2 \%)$ com a prevalência de prescrições com pelo menos dois MPIs de acordo com a lista de Beers (45,3\%), já que a metoclopramida está presente na maioria das prescrições. Importante ressaltar que alguns MPIs prescritos no contexto de internação podem de fato ser medicamentos cronicamente utilizados e seu uso pode não estar totalmente atribuível à hospitalização.

A polifarmácia, mais comumente definida como uso de 5 ou mais medicamentos, tem sido associada com risco aumentado de eventos adversos aos medicamentos, hospitalização e institucionalização em casa de repouso. ${ }^{19}$ Esta variável tem se mostrado fortemente associada ao uso de MPIs, sugerindo que o uso de vários medicamentos também pode significar exposição a substâncias onde os riscos superam os benefícios. ${ }^{11}$ Neste estudo, assim como outros estudos ${ }^{20}$, foi encontrada associação significativa entre a polifarmácia e o uso de MPI $(\mathrm{P}<0.01)$.

Estes estudo tem limitações que devem ser consideradas. Nosso estudo não teve a indicação de uso do medicamento avaliada, todos os medicamentos foram considerados, inclusive prescritos se necessário e não foi avaliado o uso da insulina de escala móvel (Critério de Beers).

\section{CONCLUSÃO}

A detecção de Medicamentos Potencialmente Inapropriados (MPI) tem sido uma estratégia utilizada para diminuir o impacto dos eventos adversos associados à complexidade dos regimes terapêuticos prescritos para a população idosa. Este estudo mostra uma grande prevalência de MPIs prescritos para idosos, necessitando de políticas de redução e racionalização da prescrição destes medicamentos. 
A atual tendência de especialização dos profissionais de saúde pode levar a uma visão parcial sobre o paciente, muitas vezes deixando de considerar suas peculiaridades fisiológicas relacionadas ao perfil etário.

Algumas estratégias podem ser colocadas em prática no âmbito hospitalar para orientar a conduta profissional como o fornecimento de informações sobre o uso inadequado de medicamentos inapropriados em idosos à equipe médica, a implantação de sistema de suporte automatizado com alertas sobre os medicamentos inapropriados e o envolvimento de equipes multidisciplinares.

Uma abordagem abrangente e multidisciplinar incluindo vários tipos de intervenções se faz necessária para melhorar a qualidade das prescrições dos idosos. O farmacêutico pode contribuir avaliando a padronização dos medicamentos na instituição, proporcionando mais opções de prescrição para a população idosa. Durante os rounds multidisciplinares, o farmacêutico clínico pode contribuir alertando sobre o uso dos MPI em idosos e as opções alternativas disponíveis para racionalizar o uso destes medicamentos.

\section{Fontes de financiamento}

Os autores declaram que a pesquisa não recebeu financiamento para a sua realização.

\section{Conflito de interesses}

Os autores declaram não possuir conflito de interesses.

\section{Colaboradores}

AHDPS Ulbrich, CT Cusinato conceberam a ideia do trabalho, analisaram os dados, interpretaram os dados e revisaram criticamente o artigo; AHDPS Ulbrich redigiu o artigo; RS Guahyba participou da coleta de dados e da revisão crítica do artigo. Todos os autores são responsáveis pela aprovação da versão final bem como são responsáveis por todos os aspectos do trabalho na garantia da exatidão e integridade de qualquer parte da obra.

\section{Agradecimentos}

Às Gerências da instituição por possibilitar a realização da pesquisa, ao Serviço de Farmácia por ceder dois auxiliares de farmácia e à Gerência de Ensino e Pesquisa da instituição pelo suporte na análise dos dados.

\section{REFERÊNCIAS BIBLIOGRÁFICAS}

1. World Health Organization - WHO. Active Ageing - A Policy Framework. Geneva: WHO, 2002.

2. Carvalho MFC, Romano-Lieber NS, Bergsten-Mendes G, et al. Polifarmácia entre idosos do Município de São Paulo - Estudo SABE. Rev Bras Epidemiol. 2012; 15(4): 817-27.

3. Gorzoni ML, Fabbri RMA, Pires, SL. Medicamentos potencialmente inapropriados para idosos. Assoc Med Bras. 2012; 58(4): 442-46.

4. Beers MH, Ouslander JG, Rollingher I, et al. Explicit criteria for determining inappropriate medication use in nursing home residents. Arch Intern Med. 1991, 151:1825-18.

5. Beers MH. Explicit criteria for determining potentially inappropriate medication use by the elderly. An update. Arch Intern Med. 1997; 157:1531-1536.

6. Fick DM, Cooper JW, Wade WE, et al. Updating the Beers Criteria for Potentially Inappropriate Medication Use in Older Adults. Arch Intern Med. 2003, 163(22): 2716-24
7. American Geriatrics Society (AGS), American Geriatrics Society Updated Beers Criteria for Potentially Inappropriate Medication Use in Older Adults. J Am Geriatr Soc. 2012; 60: 616-6.

8. Gallagher P, Ryan C, Byrne S, et al. STOPP (Screening Tool of Older Person's Prescriptions) and START (Screening Tool to Alert doctors to Right Treatment). Consensus validation. International Journal of Clinical Pharmacology and Therapeutics. 2008; 46:72-83.

9. Naugler CT, Brymer C, Stolee P, et al. Development and validation of an improving prescribing in the elderly tool. Can J Clin Pharmacol. 2000;7(2):103-7.

10. Laroche ML, Charmes JP, Merle L. Potentially inappropriate medications in the elderly: a French consensus panel list. Eur J Clin Pharmacol. 2007, 63:725-31.

11. Guaraldo L, Cano FG, Damasceno GS, et al. Inappropriate medication use among the elderly: a systematic review of administrative databases. BMC Geriatrics. 2011, 11:79.

12. Kaur S, Mitchell G, Vitetta L, et al. Interventions that can Reduce Inappropriate Prescribing in the Elderly. Drugs Aging. 2009; 26 (12): 1013-28

13. Page II RL, Linnebur SA, Bryant LL, et al. Inappropriate prescribing in the hospitalized elderly patient: Defining the problem, evaluation tools, and possible solutions. Clinical Interventions in Aging, 2010, 5: 75-87.

14. Carvalho MFC, Romano-Lieber NS, Bergsten-Mendes G, et al. Polifarmácia entre idosos do Município de São Paulo - Estudo SABE. Rev Bras Epidemiol. 2012; 15(4): 817-27.

15. Slaney H, MacAulay S, Irvine-Meek J, et al. Application of the Beers Criteria to Alternate Level of Care Patients in Hospital Inpatient Units. Can J Hosp Pharm. 2015;68(3):218-25.

16. Onda M, Imai H, Takada Y, et al. Identification and prevalence of adverse drug events caused by potentially inappropriatemedication in home bound elderly patients: a retrospective study using a nation wide survey in Japan. BMJ Open, 2015; 5: e007581.

17. Niedrig DF, Hoppe L, Mächler S, et al. Benzodiazepine Use During Hospitalization: Automated Identification of Potential Medication Errors and Systematic Assessment of Preventable Adverse Events. PLoS One, 2016; 11(10): e0163224.

18. Coutinho ESF, Silva SD. Uso de medicamentos como fator de risco para fratura grave decorrente de queda em idosos. Cad. Saúde Pública, Rio de Janeiro, 18(5):1359-1366, set-out, 2002.

19. Frazier SC. Health outcomes and polypharmacy in elderly individuals: an integrated literature review. J Gerontol Nurs, 2005;31:4-11.

20. Guimarães PL, Moura CS. Fatores Associados ao Uso de Medicamentos Impróprios de Alto Risco em Pacientes Idosos Hospitalizados. Rev Bras Farm Hosp Serv Saúde, 2012;3(4): 15-19. 